\title{
Public Lecture
}

\section{Mathematics as the Language of Nature - A Historical View N MUKUNDA* INSA-CV Raman Research Professor and Former Professor IISc, Bengaluru}

(Delivered on 29 September 2014)

\section{Introduction}

I am deeply grateful to Professor Raghavendra Gadagkar and Professor Jitendra Khurana for including me in INSA's Public Lectures Series under the Science and Society Programme. In the past, many distinguished persons have given these Lectures, it is a privilege to be in their company.

The focus in this talk will be on some aspects of the relationship between mathematics and modern science as it has developed since the $17^{\text {th }}$ century. This is a period of about 400 years, beginning with figures like Galileo and Newton. As is generally acknowledged, physics has played a special role in the growth of modern science. Moreover, among the sciences it is closest to mathematics - it is dependent on it, and also contributes to it at the deepest level. Understandably then there will be a bias towards physics in what I say. I will also bring in some philosophical ideas which hopefully will particularly interest Professors Gadagkar and Khurana.

Mathematics is much older than modern science, by about two thousand years or more. The origins of geometry and arithmetic go back to very ancient times in the earliest civilizations. Well before the appearance of modern science, mathematics was used in describing natural phenomena, for instance in astronomy. However, understandably by present day standards, we often regard those uses as comparatively simple.

In contrast, the level and sophistication of the mathematics used in the description and analysis of physical phenomena has increased dramatically as modern science has grown. Here is a famous passage from Galileo's 'Il Saggiatore' in 1623:

"Philosophy (i.e., physics) is written in this grand book - I mean the Universe - which stands continually open to our gaze, but it cannot be understood unless one first learns to comprehend the language and interpret the characters in which it is written. It is written in the language of mathematics, ..., without which it is humanly impossible to understand a single word of it; without these, one is wandering in a dark labyrinth."

Galileo is regarded as the father of modern science. Indeed Einstein (Einstein, 1933) calls him 'the father of modern physics and in fact of the whole of modern natural science'. Now compare what Galileo wrote in 1623 with Richard Feynman in his 1964 Messenger Lectures at Cornell on 'The Character of Physical Law'(Feynman, 1992):

"Every one of our laws is a purely mathematical statement in rather complex and abstruse mathematics... It gets more and more abstruse and more and more difficult as we go on ... it is impossible to explain honestly the beauties of the laws of nature in a way that people can feel, without their having some deep understanding of mathematics". 
Almost 350 years apart, yet so similar in spirit, an uncanny resemblance!

We owe many fundamental discoveries to Galileo.We also owe some basic concepts to him, let me mention just a couple. He was the first to view time as an independent variable in describing physical phenomena - all quantitative measures of phenomena can be viewed as depending on time. On the technical side, he was the first to measure time intervals of about a second reasonably accurately. Today we routinely speak of measuring time intervals down to a femto second, $10^{-15}$ seconds! Here is what Galileo said about measurement in general:

"Measure what can be measured and make measurable what cannot be measured."

Another concept he created for the description of motion of material bodies was that of acceleration. Today by familiarity we may think of this as an obvious concept, but this is not so at all. With a little bit of training, the ideas of position and speed may be grasped relatively easily. But the idea of acceleration is, as Wigner (Wigner, 1960) put it, "not a very immediate concept.' Indeed, Galileo himself said that initially he thought a useful definition would be to use the terms 'uniform acceleration' for motion in which speed increased in proportion to distance travelled, rather than to time elapsed. Only later did he adopt the definition we all learn in school.

This illustrates well a point I will return to later: the creation of concepts useful for the description and study of physical phenomena is highly nontrivial. We must appreciate that there was a time when the idea of acceleration did not exist at all. It had to be invented by Galileo who found it useful in stating the laws of freely falling bodies, and later for motion in general. Using Einstein's phrase, this was a 'free invention of the human mind' which proved extraordinarily fruitful.

\section{The Galilean - Newtonian Tradition}

Compared to Galileo's uses of mathematics, his successor Isaac Newton went much further. After all, he stood on the shoulders of giants, and was a supremely gifted mathematician. Probably his most famous discoveries in mathematics are the differential calculus, the binomial theorem for general exponents, and studies of infinite power series. His name for calculus was 'the method of fluxions', and unfortunately in this work there was a famous priority dispute with Leibnitz.

Taken together, the work of Galileo and Newton defined a new world view named after them, and it led to a pattern for natural science in which the role of mathematics is prominent. In a simplified form, this involves three key steps:

i) Careful observation of natural phenomena, if possible by controlled experiments, expressing the results in mathematical form.

ii) Based on a proposed hypothesis, derivation of predictions using mathematical analysis.

iii) Performing new observations or experiments to check the predictions.

And so on in a cyclical manner. Of course as time goes on, experimental methods and measurement accuracies continually improve. New phenomena are discovered, expanding the scope of science as a whole and of each part of it. Then new connections and theories appear, refining and replacing older ideas.

There are also very subtle aspects of this pattern that have become clear over the centuries. As I mentioned earlier, the creation of useful new concepts is highly nontrivial, they cannot be 'read off' from immediate experience in any simple manner. We will see some dramatic examples of this later. In addition, as the levels of sophistication grow, the interpretation of new experiments depends enormously on earlier theories.

\section{Two Phases in the Mathematics - Physics Relationship}

So this Galilean - Newtonian tradition is about 400 years old. Looking at developments since these beginnings, we find two qualitatively different phases in the mathematics-physics relationship. In the first phase lasting till about the 1850's, advances in mathematics and in physics went essentially in step with one another. The same gifted individuals, 
simultaneously mathematicians and physicists of the highest order, contributed to both subjects. These include Newton himself; Descartes and Fermat, Huyghens and Leibnitz; Euler, Lagrange and Laplace over the $18^{\text {th }}$ century; and Poisson, Gauss, Hamilton and Jacobi well into the 1800's. To a great extent, new ideas and methods in mathematics were inspired by and created in response to the needs of physics. Here are some familiar examples:

- $\quad$ The differential calculus, already mentioned, to describe the motion of material bodies.

- $\quad$ The extension to the theory of partial differential equations to deal with fluid dynamics, elasticity, continuous media, and later on the phenomena of electromagnetism and electromagnetic waves.

- The theory of Fourier series to deal with the problem of heat conduction, leading later to the mathematics of Fourier integrals.

There is however an outstanding exception to this general trend - the birth of the idea of a field in the 1830's due to Michael Faraday. His thinking was intensely intuitive, while his mathematical powers were limited. Based on his own and others' outstanding experimental discoveries, he 'saw' electric and magnetic 'lines of force' in space as underlying all electromagnetic phenomena. Some 30 years later Maxwell expressed Faraday's ideas in mathematical form, carried them further and thus created the classical theory of electromagnetism. On $25^{\text {th }}$ March 1857, after reading Maxwell's paper titled 'On Faraday's Lines of Force', Faraday wrote to Maxwell:

"I was at first almost frightened when I saw such mathematical force made to bear upon the subject, and then wondered to see that the subject stood it so well."

Einstein (1949) captured beautifully the parallels between the two pairs two centuries apart:

"... the pair Faraday-Maxwell has a most remarkable inner similarity with the pair Galileo-Newton - the former of each pair grasping the relations intuitively, and the second one formulating those relations exactly and applying them quantitatively."

All these successes in the first phase of about two centuries surely vindicate Galileo's declaration that mathematics is the language of Nature.

Now we turn to the second phase. Sometime in the second half of the $19^{\text {th }}$ century there came a parting of the ways, with mathematics and physics making important advances each on its own. Of course even before this mathematics had experienced many advances within itself. One important reason for this development was a change of attitude within mathematics - a realization of the need for increased rigour in the subject. An influential person in this regard was the mathematician Karl Weierstrass.

So in this second phase mathematics made striking advances in many directions. But in several instances it happened that after a few decades such independently developed mathematical ideas became crucial for describing new physical phenomena and formulating new theories. Here are three examples illustrating this (Yaglom, 1988).

\section{(a) Non-Euclidean Geometry}

Julian Schwinger (Schwinger, 1986; Schwinger, 2003) once wrote that geometry began in the mud of the Nile. Euclid's axiomatic presentation of it from around $300 \mathrm{BC}$ stood the test of time for over two thousand years. Even though the independence and consistency of the axioms were examined from time to time, it was generally believed that Euclid's geometry was the only possible consistent geometry of space. Finally in the 1830's there was a breakthrough: non Euclidean geometry is a logically consistent possibility. This advance, settling a centuries old question within mathematics, was made independently by Gauss in Germany, Lobachevsky in Russia and Bolyai in Hungary. Then in 1854 Gauss's student Bernhard Riemann gave his famous Inaugural Lecture 'On the hypotheses which lie at the foundations of geometry'. This was the beginning of Riemannian geometry.

Riemann's ideas were carried forward by a galaxy of mathematicians over about 50 years Christoffel, Ricci, Levi - Civita, Bianchi and Beltrami 
in the main. The Italian tradition in geometry is particularly strong. Their work led to a beautiful structure called the Absolute Differential Calculus. Then, in the period 1907 to 1915 when Einstein was building up his General Theory of Relativity, this whole area of mathematics was available to him to express his profound physical ideas about gravitation. The Principle of Equivalence of uniform acceleration and a constant gravitational field found a natural expression in terms of Riemannian geometry. And going on from here Einstein was able to treat the geometry of space time as non Euclidean - or better non Lorentzian and variable, and find new field equations in the framework of Riemannian geometry.

\section{(b) Non-Commutative Algebra}

The second example is the development of the ideas of matrices and higher dimensional vector spaces, and non-commutative multiplication. Some of the mathematicians involved were Hamilton, Cayley, Sylvester and Grassmann. By the early 1900's this led to the concept of infinite dimensional Hilbert spaces, and linear transformations on them. This space is a double generalization of familiar three-dimensional real Euclidean space to infinitely many dimensions and to the domain of complex numbers. Then when quantum mechanics was discovered in the mid 1920's, it soon became clear that this branch of mathematics was just the right vehicle for this development in physics.

Here is how Dirac described these two instances in his 1931 paper containing the prediction of the positron:

"Non-Euclidean geometry and non commutative algebra, which were at one time considered to be purely fictions of the mind and pastimes for logical thinkers, have now been found to be very necessary for the description of general facts of the physical world".

\section{(c) Groups and Symmetry}

The third example in this context is the creation of the concept of a group within mathematics. This began in the late $18^{\text {th }}$ century in the work of Lagrange, then in the early 1800's in the work of Galois in connection with the solution of algebraic equations of degree five. The initial development of this concept was in the finite and discrete form, and later from about the 1870 's it was generalized to the continuous version by the Norwegian mathematician Sophus Lie. It has been called the "Greatest gift of $19^{\text {th }}$ century mathematics to $20^{\text {th }}$ century physics". It is the perfect language to describe symmetry properties of physical systems and draw out their consequences.

Within physics, it was in the context of electromagnetism and special relativity that Poincaré and Einstein independently stressed the importance of symmetry properties common to many physical systems. Even Galileo's Principle of Relativity, basic to Galilean-Newtonian mechanics, is best described using Lie's theory of continuous groups. In 1918 the mathematician Emmy Noether proved a fundamental theorem connecting continuous symmetries of physical systems with corresponding conservation laws. This has become basic to the subject.

After the coming of quantum mechanics, these ideas have become much more profound and fruitful than in classical physics. This is because of the special mathematical features of the former, particularly the Superposition Principle. The two main pioneers in this work are Hermann Weyl (1950) and Eugene Wigner (1979); their ideas permeate all of modern physics including atomic, nuclear and elementary particle physics.

\section{Mathematical Formulation Before Physical Understanding}

These examples from the second phase of the interplay between mathematics and physics reinforce the feelings first expressed by Galileo. The depth of this relationship is further enhanced by a situation which has occurred often, even with Newton. I will illustrate this by recalling several examples. What has often happened is that the mathematical formulation of some new feature or law of Nature is discovered first, but the meaning or interpretation and implications are not immediately evident. These emerge rather slowly later after much effort, they may even change in the course of time. We begin with Newton. 


\section{(a) Newton's Law of Universal Gravitation}

Newton stated his Law of Universal Gravitation in the Principia in 1687. It gives the forces exerted by two point masses upon each other at a certain time at a certain distance apart:

$$
F_{12}=\text { force on } m_{1} \text { due to } m_{2}=G \cdot m_{1} m_{2} \cdot \frac{r_{2}-r_{1}}{\left|r_{2}-r_{1}\right|^{3}}
$$

Here $r_{1}$ and $r_{2}$ are the position vectors of $m_{1}$ and $\mathrm{m}_{2}$ in space at a common time. However, this idea of material bodies influencing one another across empty space - action at a distance - seemed quite strange even to Newton himself. In a letter to Richard Bentley in 1692-93 Newton wrote (Misner et al., 1973):

"That one body may act upon another at a distance through a vacuum, without the mediation of anything else, by and through which their action and force may be conveyed from one to another, is to me so great an absurdity, that I believe no man, who has in philosophical matters a competent faculty of thinking, can ever fall into."

In a famous talk in 1959 Wigner expressed it even more forcefully:

"Philosophically, the law of gravitation as formulated by Newton was repugnant to his time and to himself."

But the idea worked, it explained many observations in a unified way, and led to important predictions. Over the $18^{\text {th }}$ century the idea of action at a distance became gradually accepted, and then extended to electric and magnetic phenomena as well. Even today in fundamental physics in the nonrelativistic domain - be it chemistry or atomic or nuclear or condensed matter physics - this idea expressed through a potential is routinely used.

\section{b) Maxwell's Equations for Electromagnetism}

Maxwell's equations, built upon Faraday's insights and his own important contributions, were formulated in
1865. They unified the descriptions of electric and magnetic phenomena and optics into a grand whole, and predicted the existence of electromagnetic waves. In the three dimensional and later four dimensional forms they are:

$$
\begin{aligned}
& \underline{\nabla} \times \underline{E}=-\frac{\partial \underline{B}}{\partial t}, \underline{\nabla} \times \underline{H}=\underline{J}+\frac{\partial \underline{D}}{\partial t} \\
& \partial_{\mu} F^{\mu v}=J^{\mu}, \partial_{\lambda} F_{\mu v}+\partial_{\mu} F_{v \lambda}+\partial_{v} F_{\lambda \mu}=0
\end{aligned}
$$

Especially in the latter special relativistic language, these equations have remarkable beauty and have been the source of inspiration for later fundamental equations in physics. Maxwell himself believed that the aether, a material medium with unusual properties, was needed to carry electromagnetic waves. It was only in 1905, with the arrival of special relativity, that it was finally accepted that the aether was unnecessary. Electromagnetic fields and waves are primary entities in Nature, not made up of anything more elementary

So far this was at the classical level. About 20 years later, in 1927, Dirac applied the principles of the new quantum mechanics to Maxwell's theory. The structure of the equations was maintained, but the symbols in them became mathematical quantities of a new kind. The overall physical interpretation was completely altered, and provided the basis for the photon concept already introduced by Einstein in 1905.

Both special relativity and quantum theory were born out of Maxwell's equations. But the structure of these equations came through untouched by both revolutions, though the physical meaning was completely overhauled. No wonder that Heinrich Hertz had said of them in admiration:

"One cannot escape the feeling that these mathematical formulae have an independent existence and an intelligence of their own, that they are wiser than we are, wiser even than their discoverers, that we get more out of them than was originally put into them." 


\section{c) The Case of the Dirac Equation}

My third example is the relativistic Dirac electron wave equation, discovered by him in 1928 while trying to combine quantum mechanics and special relativity (Dirac, 1928). Pauli had already shown how to incorporate electron spin in nonrelativistic quantum mechanics, but Dirac's approach was fundamentally different and led to one of the most beautiful equations of physics. For the first time new mathematical objects called spinors were introduced into relativistic physics, and out of the equation came a series of unexpected consequences - electron spin and anomalous magnetic moment, fine structure of the hydrogen spectrum, and after many twists and turns in the interpretation the concept of the positron and antimatter.

Here is the equation written in two equivalent forms, as with Maxwell's equations:

$$
\left(\frac{c \alpha \cdot\left(\underline{p}-\frac{e}{c} \underline{A}\right)+\beta m c^{2}+e \phi}{\left(i \gamma^{\mu}\left(\partial_{\mu}+i e A_{\mu}\right)-m\right) \psi} \psi=i \hbar \frac{\partial}{\partial t} \psi ;\right. \text { (a) }
$$

As Hertz said about the Maxwell system, here too we get so much more out of this equation than was originally put in!

Quantum mechanics itself is a prime example of this situation - mathematical structure first, physical meaning later. And in this case the struggle to go from the former to the latter was enormous. Recall the transition from Schrodinger's original interpretation of his wave function to Max Born's interpretation in terms of probabilities. In one of the essays in his book "Conversations with Einstein", Heisenberg (1983) uses such phrases and sentences as these:

"... the difficulties ... concerning the problem of "physical content versus mathematical form' ... the greatest difficulties did not lie in the mathematics, but at the point where the mathematics had to be linked to nature. In the end, after all, we wanted to describe nature, and not just do mathematics".

\section{Some Significant Lessons}

These instances of mathematical formulation preceding physical understanding highlight some significant features of our subject which we should recognize. Let me describe them briefly.

a) One of Newton's famous statements was 'hypotheses non fingo' 'I do not frame hypotheses'. However, as Einstein says (1933):

"Newton ... still believed that the basic concepts and laws of his system could be derived from experience; his phrase 'hypotheses non fingo' can only be interpreted in this sense".

The implication was that the concepts needed for the formulation of physical laws could be 'read off' more or less easily from direct experience. While this may have seemed to be so four centuries ago, it is certainly far from the truth in later times. Actually even for Galileo, as we saw, the most useful definition of acceleration was not obvious at first sight. Newton himself was uneasy with concepts like absolute space, absolute rest, and action at a distance.

We know that the concept of the electromagnetic field - a constituent of Nature in addition to matter - took many years to crystallize, starting with Faraday's intuitive ideas and followed by Maxwell's mathematical formulation. In the case of general relativity, which identified the metric of space time as the field representing gravitation, many steps were involved - the transition from Newtonian to special relativistic space time; Einstein's profound analysis of the Principle of Equivalence; and the realization of the need to generalize the Lorentz geometry of special relativity to a more general Riemannian structure (Schwinger, 1986; Schwinger, 2003). So in both cases the passage from 'direct experience' to fundamental new concepts was hardly immediate.

In the quantum realm, the discoveries of Schrodinger's and Dirac's wave equations are really very far removed from immediate experience, indeed their wave functions are not even directly measurable. The effort needed to go from experience to new 
concepts in physics has increased enormously. We are reminded of Einstein's widely quoted remark from 1933:

"... the axiomatic basis of theoretical physics cannot be abstracted from experience but must be freely invented ...".

b) The examples of the previous Section suggest the following: whenever the mathematical form of a new law of physics is found, we seem to come into direct contact with Nature, 'touching' or 'capturing' physical reality. This point has been eloquently expressed by Wigner and by Heisenberg:

Wigner (1960): “... the mathematical formulation of the physicist's often crude experience leads in an uncanny number of cases to an amazingly accurate description of a large class of phenomena. This shows that the mathematical language has more to commend it than being the only language which we can speak: it shows that it is, in a very real sense, the correct language".

Heisenberg (1971): "If nature leads us to mathematical forms of great simplicity and beauty that no one has previously encountered, we cannot help thinking that they are 'true', that they reveal a genuine feature of nature." this talk!

Such statements resonate well with the title of

c) A third qualifying point must now be made: the considerable effort needed to interpret physically a new mathematical formulation is accompanied by the fact that such interpretation is always evolving and never final. To go from Newton's action at a distance form of gravitation to the local field form given by general relativity took about 230 years, and it is not yet the 'final' word. Similarly in the case of light we have moved from the classical picture in Maxwell's treatment to the photon concept in quantum theory, then on to quantum electrodynamics, itself now part of a larger theory.

\section{On the Natures of Mathematics and Mathematical Knowledge}

At this point let us turn to the questions - what can we say about the nature of mathematics itself, and about our knowledge of mathematics?

Within mathematics there has been a debate for a long time between two schools of thought - the 'realists' or 'Platonists', and the 'constructivists' (Changeux and Connes, 1995). The realists believe that mathematical structures and truths 'exist somewhere' on their own, making up a whole 'continent of mathematics'. We only keep uncovering it a little bit at a time, like explorers. Prominent in this group are Descartes, Newton, Leibnitz, Hermite, Cantor, Godel and Hardy. The constructivists on the other hand view mathematics as a purely human creation in response to our experiences and needs. In this group we find Kronecker, Poincaré and Brouwer.

These are opposed points of view, and neither side can convince or convert the other. So they may seem to be essentially expressions of personal preference and psychology. But let us keep these ideas in mind as we go further. If we view mathematics as a part of human language, of our means of communication, we recognize that it has some very special qualities - it can achieve very high levels of precision and compression of content. It also allows for tremendously efficient manipulations in drawing out the consequences of given assumptions.

Let us now move closer to the sciences and bring in some ideas of a philosophical nature. I described earlier the Galilean - Newtonian tradition in physics. During the $18^{\text {th }}$ century, it was spectacularly successful in many areas such as mechanics, hydrodynamics and astronomy. Towards the end of the century, the discoveries in electrostatics and magnetostatics also fell into this pattern.

Around this time, the philosopher Immanuel Kant tried to explain why the Galilean - Newtonian approach to understanding Nature was so successful. He developed a profound theory of the nature of human knowledge, bringing together the rationalist and empiricist schools of philosophy. Kant made a 
distinction between a priori and a posteriori forms of knowledge - that which we possess at birth in advance of experience, and that which we acquire through experience. Many concepts underlying Galilean Newtonian physics were supposed to be a priori, built into our minds in advance of experience - Euclidean geometry of space, simultaneity of events in time, determinism, the permanence of objects, the law of mass conservation, and even Newton's Third Law of Motion.

Later progress during the $19^{\text {th }}$ century and beyond showed that this could not be entirely correct. Many of the Kantian a prioris - separate and absolute space and time, absoluteness of simultaneity, determinism, mass conservation, even Euclidean geometry - had to be gradually revised. At a deeper level the question remained - what is the origin of the a priori component of knowledge, and how is it able to handle later sensory experiences so well?

Naturally, over the years many mathematicians and physicists responded to Kant's ideas in their own ways. I will restrict myself to only two of them. In a 1930 lecture the mathematician David Hilbert said (Reid, 1972):

“... the most general basis of Kant's theory of knowledge retains its importance. ... But the line between that which we possess a priori and that for which experience is necessary must be drawn differently ... Kant has greatly overestimated the role and the extent of the a priori ... Kant's a priori theory contains anthropomorphic dross from which it must be freed. After we remove that, only that a priori will remain which also is the foundation of pure mathematical knowledge."

Einstein, in comparison, was quite negative:

"I am convinced that the philosophers have had a harmful effect upon the progress of scientific thinking in removing certain fundamental concepts from the domain of empiricism where they are under our control, to the intangible heights of the a priori."

Returning to the question of the origin of the Kantian a priori, a fascinating answer was given by the biologist Konrad Lorenz (1966) in the 1940's. This was later beautifully elaborated by Max Delbruck (1986) in the 1970's. It is based on the theory of Darwinian evolution guided by natural selection, and the role of 'phylogenetic learning'. This is a very slow learning process involving the species as a whole over many generations, through interaction with its environment and phenomena at roughly its own scales of length, mass and time. This part of Nature is called the 'World of Middle Dimensions', and developing the capacities to pick out its most important features is beneficial to survival.

Contrasted with this slow learning process is 'ontogenetic learning', the experiences of each individual from birth to death over an individual life span. The capacities created by species evolution guided by natural selection are then given ready made at birth to each individual member of the species, an inheritance you might say, in advance of experience. Quoting from Delbruck (1986):

"... two kinds of learning are involved in our dealing with the world.One is phylogenetic learning ... during evolution we have evolved very sophisticated machinery for perceiving and making inferences about a real world ... . What is a priori for the individual is a posteriori for the species. The second kind of learning involved in dealing with the world is ontogenetic learning, namely the lifelong acquisition of cultural, linguistic and scientific knowledge."

Recall now that Hilbert's ideas were expressed some years before Lorenz (and Delbruck) gave their explanation of the a priori component of human knowledge. Putting the two sets of ideas together, we are naturally led to ask: Is the capacity of the human mind for mathematical thinking also a result of biological evolution, a result of phylogenetic learning, to respond to the fact that the laws of Nature are 
coded in mathematics? We are encouraged here by this sentence from a talk in 1939 by Dirac (1939) (in the spirit of Galileo) that there is

"... some mathematical quality in Nature, a quality which the casual observer of Nature would not suspect, but which nevertheless plays an important role in Nature's scheme".

So let me repeat for clarity: here we have

(i) Hilbert saying that the only a priori component of human knowledge is the foundation of mathematics;

(ii) Dirac pointing out an unsuspected mathematical quality in Nature; and

(iii) Lorenz and Delbruck explaining that all a priori knowledge comes from phylogenetic learning, species interacting with the World of Middle Dimensions within Nature.

Granted that it may seem very speculative, may we not then say that over evolutionary time we have acquired a capacity and sensitivity to this subtle aspect of Nature, a sixth sense? But in contrast to the other contents of Nature which are accessible to our other senses and instruments, this mathematical quality can be 'seen' only by the mind and not by the eye. This reminds us of the difference between our biologically acquired responses to space and to time, the latter being much more 'mental'. Going back to Kant again, he had called space and time the external and the internal forms of intuition. In a way, it, i.e. mathematics, is similar to the human capacity for language; but as with language, here too the subsequent 'cultural' enlargement of mathematical ideas has led to a world of its own.

\section{Some Concluding Thoughts}

I have traced the role of mathematics in physical science, and the relation between them, in a historical vein and from several points of view. It is no surprise that this relationship has deepened and grown enormously in sophistication, since the beginnings of modern science. Yet the situation is probably far more complex and subtle than we might have anticipated.
With respect to the realist-constructivist debate within mathematics, we are apparently in a somewhat intermediate position - it is objectively true that Nature expresses its laws in the language of mathematics, but the world of mathematics contains much more than seems necessary to describe Nature. This reminds us of CN Yang's (1981) statement comparing mathematics and physics, on the occasion of the Einstein centennial in 1979:

"At the fundamental conceptual level they amazingly share some concepts, but ... the life force of each discipline runs along its own veins."

At the end, acknowledging the mathematical quality of Nature as an objective fact, one may still ask - why is it so? To this, as far as I know, there is no convincing answer. I can only repeat what the Masters have said on this question, the first of them in a somewhat playful manner:

Wigner (1960): "The miracle of the appropriateness of the language of mathematics for the formulation of the laws of physics is a wonderful gift which we neither understand nor deserve."

Einstein $(1936 ; 1950 ; 2005)$ : “The eternal mystery of the world is its comprehensibility ... . The fact that it is comprehensible is a miracle."

I hope you find what I have presented to be interesting, and will feel motivated to think further on these matters.

My thanks again to Professor Gadagkar, Professor Khurana, Shri Sahni for giving me this opportunity to speak, and fine hospitality.

\section{Acknowledgements}

The ideas presented in this talk overlap significantly with an earlier talk given 28 February 2014 on "Role of Mathematics in Science through History" at the Nehru Science Centre, Mumbai. I thank Dr Arvind Paranjpye and Shri Satish Sahney for graciously allowing me to present these ideas again, with some variations, to a different audience. 


\section{References}

Changeux JP and Connes A (1995) Conversations on Mind, Matter and Mathematics. Princeton University Press

Delbruck Max (1986) Mind from Matter? An Essay on Evolutionary Epistemology. Blackwell Scientific

Dirac PAM (1931) Quantised Singularities in the Electromagnetic Field, Proc Roy Soc (London) A133 60

Dirac PAM (1928) The Quantum Theory of the Electron I, II Proc Roy Soc (London) A117 610; A118 351. A historical and pedagogical account is given in Sin-ItiroTomonaga, The Story of Spin, University of Chicago Press (1997)

Dirac PAM (1939) The Relation between Mathematics and Physics Proc Roy Soc (Edinburgh) 59 122-129

Einstein A (2005) Physics and Reality, 1936 essay in Out of my later years, first published 1950. Castle Books

Einstein A (1949) Autobiographical Notes in Albert Einstein Philosopher Scientist (Eds: Schilpp PA), Vol. VII in the Library of Living Philosophers, Open Court Publishing Company, Le Salle, Illinois

Einstein A (1933) On the Method of Theoretical Physics Herbert Spencer Lecture, Oxford

Feynman Richard P(1992) The Character of Physical Law. Penguin Books, 39

Galilei Galileo, Il Saggiatore (in Italian), Rome, 1623 (1960) The Assayer, English translation by Stillman Drake and C. D. OMalley. University of Pennsylvania Press

Heisenberg W (1971) Physics and Beyond - Encounters and Conversations, George Allen \& Unwin, Ltd, 68
Heisenberg W (1983) The Beginnings of Quantum Mechanics in Göttingen, 48 in Encounters with Einstein and other Essays on People, Places and Particles. Princeton University Press

Lorenz Konrad (1966) The Natural Science of the Human Species: An Introduction to Comparative Behavioral Research The Russian Manuscript, 1944-48. (ed. Agnes von Cranach) transl. Robert D. Martin, M.I.T. Press

Misner CW, Thorne KS and Wheeler JA (1973) Gravitation. W. H. Freeman \& Co. San Francisco, 40-41

Noether E (1918) Invariante Variations probleme, Nachr. d. König. Gesellsch. D. Wiss. zuGöttingen. Math-phys Klasse 235257

Reid Constance (1972) Hilbert. Springer, New York, 190

Schwinger J (2003) Einsteins Legacy - The Unity of Space and Time, Scientific American, New York (1986); Dover Publications

Very readable accounts of all these may be found in I. M. Yaglom, Felix Klein and Sophus Lie - Evolution of the Idea of Symmetry in the Nineteenth Century, Birkhauser, Boston (1988)

Weyl H (1950) The Theory of Groups and Quantum Mechanics, Dover

Wigner EP (1960) The Unreasonable Effectiveness of Mathematics in the Natural Sciences Communications in Pure and Applied Mathematics $\mathbf{1 3}$

Wigner EP (1979) Symmetries and Reflections - Scientific Essays, Ox Bow Press, Wood bridge, Connecticut, USA

Yang CN (1981) To Fulfill a Vision - Jerusalem Einstein Centennial Symposium (ed. Y. Neeman), Addison-Wesley. 